\title{
Nipple-Areola Complex Necrosis after Nipple- Sparing Mastectomy with Immediate Autologous Breast Reconstruction
}

\author{
Jin-Woo Cho, Eul-Sik Yoon, Hi-Jin You, Hyon-Surk Kim, Byung-Il Lee, Seung-Ha Park \\ Department of Plastic and Reconstructive Surgery, Korea University College of Medicine, Seoul, Korea
}

Background Autologous or implant-based breast reconstruction after nipple-sparing mastectomy is increasingly preferred worldwide as a breast cancer treatment option. However, postoperative nipple-areola complex (NAC) necrosis is the most significant complication of nipplesparing mastectomy. The purpose of our study was to identify the risk factors for NAC necrosis, and to describe the use of our skin-banking technique as a solution.

Methods We reviewed cases of immediate autologous breast reconstruction after nipplesparing mastectomy at our institution between June 2005 and January 2014. The patients' data were reviewed and the risk of NAC necrosis was analyzed based on correlations between patient variables and NAC necrosis. Moreover, data pertaining to five high-risk patients who underwent the donor skin-banking procedure were included in the analysis.

Results Eighty-five patients underwent immediate autologous breast reconstruction after nipple-sparing mastectomy during the study period. Partial or total NAC necrosis occurred in 36 patients (43.4\%). Univariate analysis and binary regression modeling found that body mass index, smoking history, radiation therapy, and mastectomy volume were significantly associated with NAC necrosis. Of the 36 cases of NAC necrosis, 31 were resolved with dressing changes, debridement, or skin grafting. The other five high-risk patients underwent our prophylactic skin-banking technique during breast reconstruction surgery.

Conclusions NAC necrosis is common in patients with multiple risk factors. The use of the skin-banking technique in immediate autologous breast reconstruction is an attractive option for high-risk patients. Banked skin can be used in such cases without requiring additional donor tissue, with good results in terms of aesthetic and reconstructive outcomes.

Keywords: Surgical flaps / Nipples / Necrosis / Risk factors
Correspondence: Eul-Sik Yoon Department of Plastic and Reconstructive Surgery, Korea University College of Medicine, 73 Inchon-ro, Seongbuk-gu, Seoul 02841 , Korea

Tel: +82-2-920-5368

Fax: + 82-2-922-7437

E-mail: yesanam2@korea.ac.kr
This article was presented as a poster at the Twelfth Korea-Japan Congress of Plastic and Reconstructive Surgery on May 15-17, 2014, Incheon, Korea.

No potential conflict of interest relevant to this article was reported.

Received: 4 Mar 2015 • Revised: 22 May $2015 \bullet$ Accepted: 1 Jun 2015

pISSN: 2234-6163 • elSSN: 2234-6171 • http://dx.doi.org/10.5999/aps.2015.42.5.601 • Arch Plast Surg 2015;42:601-607

\section{INTRODUCTION}

Recently, autologous or implant-based breast reconstruction after nipple-sparing mastectomy (NSM) has been increasingly preferred worldwide as a breast cancer treatment option [1-5]. Multiple oncological studies have established that NSM leads to a reduced risk of local recurrence [6,7]. However, despite its effectiveness and aesthetic superiority, NSM has been associated with several complications, with postoperative nipple-areola complex (NAC) necrosis being the main concern [2,8-11].

Resecting the tissue located below the NAC causes injury to local perforators and leads to a risk of ischemia in the NAC $[8,12]$. 
Once NAC necrosis occurs after NSM, surgical procedures are often performed to repair the defects. The use of conventional local flaps or skin grafts may pose aesthetic and donor site problems, and may cause anxiety in patients. Given these problems, the use of donor skin that is temporarily banked behind the patient's own NAC is an alternative solution for replacing necrotic tissue in cases of uncertain viability.

Since the introduction of the skin-banking technique, it has been used with some frequency [13-17]. However, to the best of our knowledge, the risk factors for this procedure and its outcomes have been not been previously studied with an algorithmic approach. In this study, we examined the risk factors for NAC necrosis, performed the prophylactic skin-banking technique in high-risk patients, and achieved relatively good results and patient satisfaction outcomes.

\section{METHODS}

We conducted a retrospective review of the medical charts of 85 patients who underwent immediate autologous breast reconstruction after NSM at our institution between June 2005 and January 2014. The majority of the study group comprised patients who underwent autologous tissue reconstruction only, as well as some patients who underwent implantation in combination with autologous reconstruction.

Only patients without any evidence of preoperative cancer invasion of the NAC underwent NSM. NSM was performed using inframammary, radial, and lateral incisions either with or without a periareolar incision. Frozen samples of all subareolar tissue were pathologically examined intraoperatively. When subareolar involvement was confirmed, the NAC was sacrificed. When the involvement was equivocal, an additional frozen biopsy was performed. The intraoperative frozen biopsy result determined whether the NAC would be sacrificed.

All autologous reconstructions were performed by the senior author of this study (E.S.Y.) after NSM was performed by two experienced general breast surgeons. The autologous reconstruction procedure was performed using conventional methods, including pedicled or free transverse rectus abdominis myocutaneous (TRAM) flaps and latissimus dorsi myocutaneous flaps. The method was determined according to the patient's condition and the surgeon's preference.

The flaps were harvested using the conventional method, and all skin was de-epithelialized and used to fill the breast volume. However, five patients with a high risk of nipple necrosis underwent the prophylactic donor skin-banking procedure in order to preemptively address the possibility of necrosis. A circular remnant of the skin of the donor flap, approximately $5 \mathrm{~cm}$ in diame- ter, was left and buried under the patient's breast envelope at a location symmetric to the contralateral nipple. When NAC necrosis was observed postoperatively, the banked skin was pulled out and used as the skin envelope of the breast. Approximately three to six months later, the NAC was reconstructed under local anesthesia. When the NAC was intact, the banked skin was de-epithelialized and used to fill the internal volume of the breast approximately two weeks after the main reconstruction.

The patients' data were reviewed to assess possible risk factors for NAC necrosis. The patients' general demographic characteristics and medical charts, including underlying systemic diseases such as diabetes mellitus, liver and kidney disease, and heart failure, were examined to identify risk factors that appeared in the preoperative evaluation. In addition, information about the incisions made during NSM, mastectomy volume, and intraoperative pathological findings from frozen subareolar tissue samples was obtained based on the operative records of each patient. Finally, postoperative progress records, final oncological pathological findings, and preoperative and postoperative clinical photographs were reviewed. These variables were then analyzed to determine whether they showed a correlation with NAC necrosis. NAC necrosis was defined as full-thickness necrosis, requiring surgical procedures such as debridement, delayed repair, and skin grafting.

The data were analyzed using SPSS ver. 22.0 (IBM Co., Armonk, NY, USA). The chi-squared test or Fisher's exact test was performed to estimate the correlations among the categorical risk factors and outcomes. Binary logistic regression analysis was also performed to examine the adjusted odds ratios of nipple necrosis for each risk factor. P-values $<0.05$ were considered to indicate statistical significance.

\section{RESULTS}

Eighty-five patients underwent immediate autologous breast reconstruction after NSM during our study period. Their mean age was 44.3 years and their mean body mass index (BMI) was $23.4 \mathrm{~kg} / \mathrm{m}^{2}$. Nine patients had a history of smoking, and $18 \mathrm{had}$ underlying systemic disease. A total of 46 patients underwent NSM in the right breast, 35 patients in the left breast, and four patients in both breasts. Partial or total NAC necrosis occurred in 36 patients (43.4\%) (Table 1).

We examined the variables related to patient data through univariate analysis (Table 2). In the older and younger patient groups, 30 patients (46.2\%) and six patients (20\%) experienced NAC necrosis, respectively. However, this difference was not significant $(\mathrm{P}=0.301)$. Twenty-two patients were classified as obese $\left(\mathrm{BMI} \geq 25 \mathrm{~kg} / \mathrm{m}^{2}\right)$, and 63 patients were classified as having a 
Table 1. Summary of the demographic characteristics and clinical data of the 85 patients enrolled in this study

\begin{tabular}{|lc|}
\hline Variable & Value (\%) \\
\hline Age (yr) & $44.3 \pm 8.0$ (mean \pm standard deviation) \\
$<40$ & $20(23.5)$ \\
$\geq 40$ & $65(76.5)$ \\
Body-mass index $\left(\mathrm{kg} / \mathrm{m}^{2}\right)$ & $23.4 \pm 2.9$ (mean \pm standard deviation) \\
$<25$ & $63(74.1)$ \\
$\geq 25$ & $22(25.9)$ \\
Smoking & \\
Ex-smoker & $2(2.4)$ \\
Smoker & $7(8.2)$ \\
None & $76(89.4)$ \\
Underlying disease & \\
Diabetes mellitus & $4(4.7)$ \\
Hypertension & $13(15.3)$ \\
Kidney disease & $3(3.5)$ \\
Heart disease & $2(2.4)$ \\
Others & $5(5.9)$ \\
None & $67(78.8)$ \\
Laterality & $46(54.1)$ \\
Right & $35(41.2)$ \\
Left & $4(4.7)$ \\
Bilateral & \\
Nipple-areola complex necrosis & $49(57.6)$ \\
None & $26(30.6)$ \\
Partial & $10(11.8)$ \\
Total & \\
\hline & \\
& \\
\hline &
\end{tabular}

Table 2. Results of the univariate analysis of the patientrelated risk factors for nipple-areola complex necrosis

\begin{tabular}{|c|c|c|c|}
\hline Risk factor & NAC necrosis & NAC intact & P-value \\
\hline Age (yr) & & & 0.301 \\
\hline$<40$ & 6 & 14 & \\
\hline$\geq 40$ & 30 & 35 & \\
\hline Body mass index $\left(\mathrm{kg} / \mathrm{m}^{2}\right)$ & & & 0.025 \\
\hline$<25$ & 22 & 41 & \\
\hline$\geq 25$ & 14 & 8 & \\
\hline Smoking history & & & 0.033 \\
\hline Yes & 7 & 2 & \\
\hline No & 29 & 47 & \\
\hline Underlying disease & & & 0.592 \\
\hline Yes & 9 & 9 & \\
\hline No & 27 & 40 & \\
\hline Preoperative radiation therapy & & & 0.038 \\
\hline Yes & 6 & 1 & \\
\hline No & 30 & 48 & \\
\hline Preoperative chemotherapy & & & 0.311 \\
\hline Yes & 6 & 4 & \\
\hline No & 30 & 45 & \\
\hline Previous breast surgery & & & 0.380 \\
\hline Yes & 7 & 6 & \\
\hline No & 29 & 43 & \\
\hline Total number & 36 & 49 & - \\
\hline
\end{tabular}

normal BMI. NAC necrosis was more likely in the overweight or obese group than in the non-overweight group (63.6\% vs. $34.9 \%, \mathrm{P}=0.025)$. NAC necrosis occurred more frequently in
Table 3. Results of the univariate analysis of the operative and oncological risk factors for nipple-areola complex necrosis

\begin{tabular}{|c|c|c|c|}
\hline Risk factor & NAC necrosis & NAC intact & P-value \\
\hline Incision & & & 0.179 \\
\hline Including periareolar & 34 & 41 & \\
\hline Excluding periareolar & 2 & 8 & \\
\hline Reconstruction & & & 0.143 \\
\hline LD \pm implant & 9 & 24 & \\
\hline TRAM & 26 & 21 & \\
\hline Others & 1 & 4 & \\
\hline Sub-areolar atypical cell & & & 0.727 \\
\hline Yes & 3 & 6 & \\
\hline No & 33 & 43 & \\
\hline Pathological finding & & & 0.447 \\
\hline Carcinoma in situ & 29 & 35 & \\
\hline Invasive carcinoma & 7 & 14 & \\
\hline Lymph node metastasis & & & 0.825 \\
\hline Yes & 13 & 19 & \\
\hline No & 23 & 30 & \\
\hline Mastectomy volume (g) & & & 0.028 \\
\hline$<200$ & 6 & 15 & \\
\hline $200-400$ & 15 & 26 & \\
\hline$>400$ & 15 & 8 & \\
\hline Total number & 36 & 49 & - \\
\hline
\end{tabular}

Table 4. Significant risk factors for nipple-areola complex necrosis in the binary regression model

\begin{tabular}{|lrcc|}
\hline Variable & $\begin{array}{c}\text { Odds } \\
\text { ratio }\end{array}$ & $\begin{array}{c}\text { 95\% Confidence } \\
\text { interval }\end{array}$ & P-value \\
\hline Body-mass index $\left(\mathrm{kg} / \mathrm{m}^{2}\right)$ & 2.31 & $0.71-7.46$ & 0.163 \\
Smoking history & 3.92 & $0.63-24.59$ & 0.145 \\
Preoperative radiation therapy & 10.92 & $1.02-117.22$ & 0.048 \\
Incision & 2.59 & $0.45-15.04$ & 0.290 \\
Mastectomy volume $(\mathrm{g})$ & 2.25 & $1.01-5.03$ & 0.048 \\
\hline
\end{tabular}

patients with a history of smoking than in non-smokers $(77.8 \%$ vs. $38.2 \%, \mathrm{P}=0.033)$. A preoperative history of radiation therapy was significantly associated with NAC necrosis ( $85.7 \%$ vs. $38.5 \%, \mathrm{P}=0.038$ ). The variables relating to systemic underlying disease, chemotherapy, and previous breast operations were not significantly associated with NAC necrosis.

Univariate analysis was used to examine the operative and oncological risk factors (Table 3). Mastectomy volumes $>400 \mathrm{~g}$ were significantly associated with the occurrence of NAC necrosis. However, the presence of a periareolar incision and the reconstruction methods used during the operation were not significantly associated with the occurrence of NAC necrosis $(\mathrm{P}=0.179$ and $\mathrm{P}=0.143$, respectively). The intraoperative biopsy results of the frozen subareolar tissues, the final pathological results, and lymph node metastasis were likewise not significantly associated with NAC necrosis $(\mathrm{P}=0.727, \mathrm{P}=0.447$, and $\mathrm{P}=0.825$, respectively). 
Table 5. Demographic characteristics and clinical data of five patients who underwent the skin-banking technique

\begin{tabular}{|lccccccccc}
\hline Case & $\begin{array}{c}\text { Age } \\
(\mathbf{y r})\end{array}$ & $\begin{array}{c}\text { BMI } \\
\left(\mathbf{k g} / \mathbf{m}^{2}\right)\end{array}$ & Smoking & $\begin{array}{c}\text { Radiation } \\
\text { history }\end{array}$ & $\begin{array}{c}\text { Underlying } \\
\text { disease }\end{array}$ & $\begin{array}{c}\text { Pathologic } \\
\text { finding }\end{array}$ & $\begin{array}{c}\text { Mastectomy } \\
\text { volume (g) }\end{array}$ & $\begin{array}{c}\text { Recons- } \\
\text { truction }\end{array}$ & $\begin{array}{c}\text { NAC } \\
\text { necrosis }\end{array}$ \\
\hline 1 & 46 & 19.83 & Smoker & None & None & DCIS & 150 & MS-II TRAM & Total \\
2 & 40 & 27.76 & Non-smoker & None & None & Invasive ductal carcinoma & 530 & MS-II TRAM & Partial \\
3 & 54 & 21.47 & Non-smoker & None & None & Invasive lobular carcinoma & 260 & Pedicled LD & Partial \\
4 & 47 & 28.10 & Ex-smoker & None & HTN, DM & Invasive ductal carcinoma & 545 & MS-II TRAM & Total \\
5 & 38 & 25.99 & Non-smoker & Positive & None & Invasive ductal carcinoma & 522 & Robot-assisted & Partial \\
& & & & & & & & LD & \\
\hline
\end{tabular}

BMI, body mass index; NAC, nipple-areola complex; DCIS, ductal carcinoma in situ; MS-II TRAM, muscle-sparing transverse rectus abdominis musculocutaneous; LD, latissimus dorsi; HTN, hypertension; DM, diabetes mellitus.

\section{Fig. 1. A case in which the banked skin was pulled out and used}

A 46-year-old patient underwent immediate breast reconstruction with a transverse rectus abdominis myocutaneous flap. Since the first intraoperative frozen section of the subareolar tissue was positive for malignant cells, additional subareolar tissue was excised. (A) Preoperative clinical photographs. (B) Total nipple-areola complex (NAC) necrosis occurred on the eleventh postoperative day. We decided to use banked skin to cover the defect. (C) A clinical photograph obtained four months postoperatively after the banked skin was pulled out. (D) A clinical photograph obtained 30 months after NAC reconstruction with tattooing.
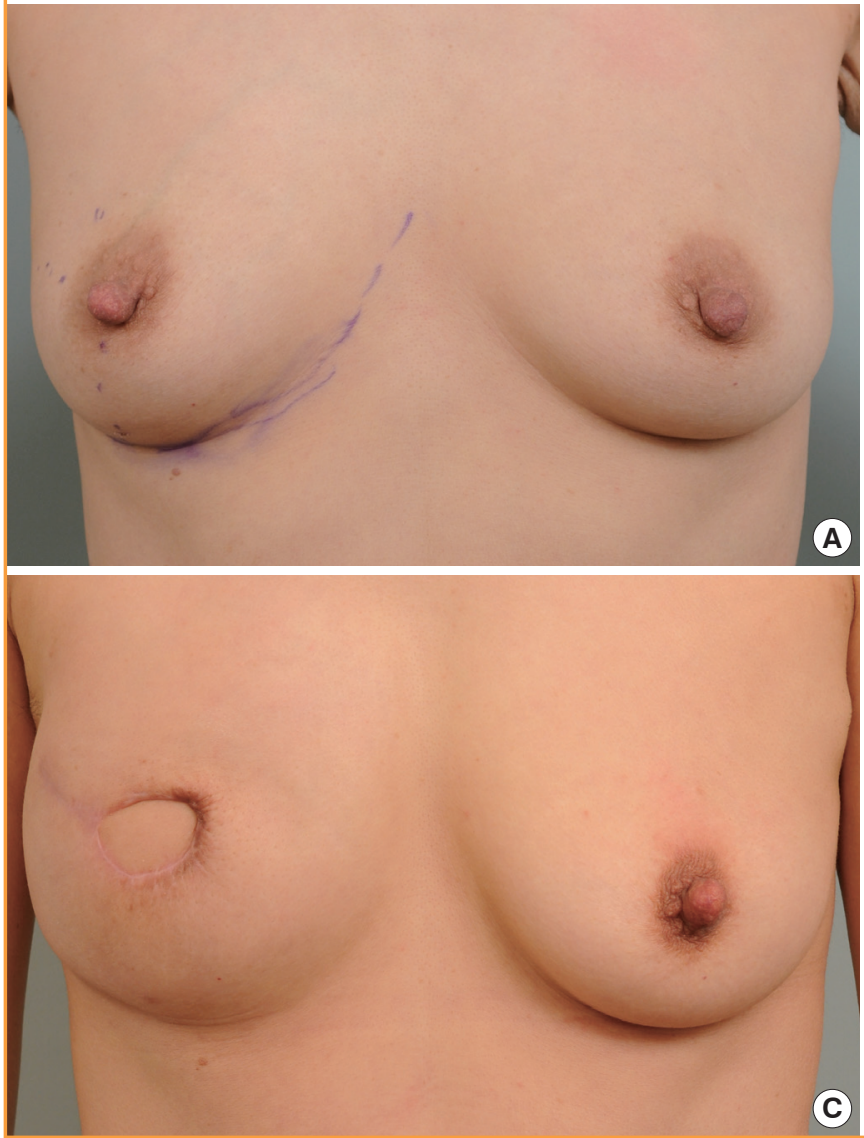

Regression analysis was used to further assess the potential contribution of the above variables to the development of NAC necrosis. The variables included were BMI, smoking, radiation therapy, incision method, and mastectomy volume. In a binary regression model, radiation therapy and mastectomy volume were found to be independent risk factors for the development of NAC necrosis (Table 4). The Hosmer-Lemeshow test sug-
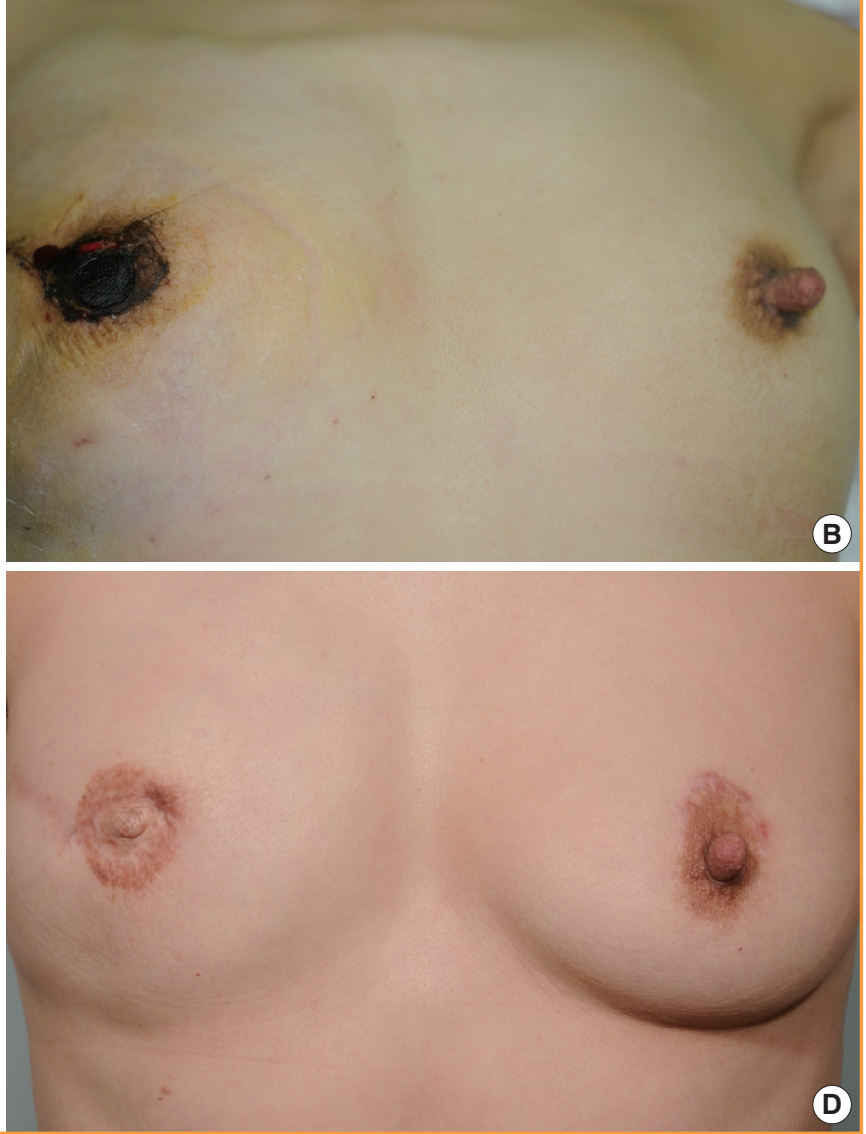

gested a good fit $(\mathrm{P}=0.718)$ for the regression model.

Of the 36 cases of NAC necrosis, 31 cases were resolved with secondary intention, debridement of the necrosis, or a conventional skin graft. Among the remaining five patients, four who were at a high risk of developing NAC necrosis underwent prophylactic skin-banking during breast reconstruction surgery (Table 5). The skin-banking group had at least one significant 


\section{Fig. 2. A case in which the banked skin was buried}

A 40-year-old patient underwent immediate breast reconstruction with transverse rectus abdominis myocutaneous flap coverage after nipplesparing mastectomy. Vertical-reduction pattern mastopexy was performed simultaneously with the reconstruction. (A) An intraoperative photograph of skin banking. (B) Immediate postoperative findings, with a small window exposing the banked skin. (C) The partial nipple-areola complex necrosis that occurred after two weeks. The banked skin was de-epithelialized and buried under the native nipple-areola complex. (D) A clinical photograph obtained five months postoperatively, after excision of the necrotic skin with delayed repair.
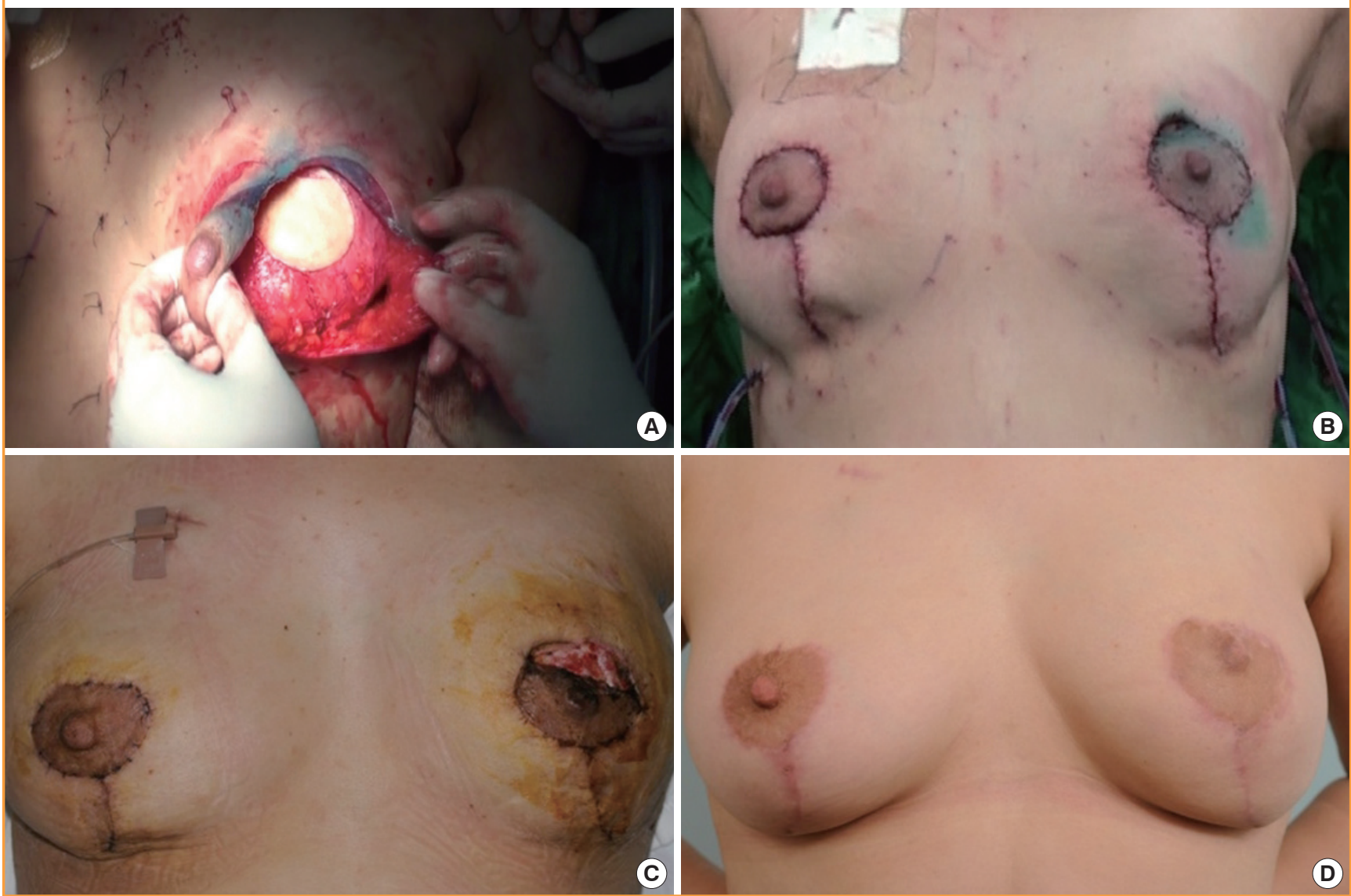

risk factor for NAC necrosis or intraoperative ischemia of the NAC (case 3 in Table 5). In two of the five patients who underwent skin-banking, the banked skin was used to replace the tissue with total NAC necrosis (case 1, Fig. 1). In the other patients (the partial necrosis group), the banked skin was not used and was de-epithelialized (case 2, Fig. 2). After complete wound healing, nipple reconstruction or correction was performed as needed, approximately five months postoperatively.

\section{Case 1}

A 46-year-old patient underwent immediate breast reconstruction with a TRAM flap. She was a smoker, and additional subareolar tissue was excised because the first intraoperative frozen section of the subareolar tissue was positive for atypical cells. Total NAC necrosis occurred on the eleventh postoperative day. We decided to use banked skin to cover the defect. Fig. 1 shows the preoperative and postoperative clinical photographs of the $\mathrm{NAC}$ reconstruction with tattooing.

\section{Case 2}

A 40-year-old patient underwent immediate breast reconstruction with TRAM flap coverage after NSM. Vertical-reduction pattern mastopexy was performed simultaneously with reconstruction. Partial NAC necrosis occurred two weeks after the reconstruction. The banked skin was de-epithelialized and buried under the native NAC. Fig. 2 shows the preoperative and postoperative clinical photographs of the de-epithelialized and buried banked skin with delayed repair.

\section{DISCUSSION}

Several oncological studies have established that NSM is associated with a reduced risk of local recurrence [6,7]. Many studies have recommended that NSM only be performed in patients with peripheral tumors located no closer than $5 \mathrm{~cm}$ to the NAC [1]. However, the indications for NSM are currently being broadened. Intraoperative biopsy of the subareolar tissue or intraoper- 
ative irradiation of the nipple is performed to reduce the risk of local recurrence [1].

Several studies have described NAC necrosis after NSM. The reported incidence of NAC necrosis has been reported to range from $0 \%$ to $48 \%[2,3,8-10,18,19]$. In our study, the incidence of NAC necrosis was $43.4 \%$. The relatively high incidence of NAC necrosis in our study was partly a result of our definition of necrosis. In addition to including both cases of partial and total necrosis, our definition of partial and total NAC necrosis was relatively unusual; we defined it as full-thickness necrosis requiring surgical intervention such as debridement, delayed repair, and skin grafting. Moyer et al. [8] and Carlson et al. [9], on the other hand, defined nipple necrosis as any nipple ischemia requiring local wound care. Moreover, Dent et al. [19] stratified NAC necrosis into three subgroups according to the thickness of the involved tissue. Some degree of bias may exist due to the lack of standard criteria for treating NAC ischemia after NSM. Even small defects have a significant impact on the final aesthetic result of breast reconstruction. Another reason for inconsistent results is the extent to which the subareolar tissue is resected. In our institution, radical resection of the subareolar tissue using a periareolar incision was performed in order to ensure oncological safety. Previous studies have indicated that the periareolar incision pertains the highest risk for NAC necrosis. Several studies have evaluated the risk factors of NAC necrosis according to the surgical method employed $[5,8-10,19,20]$. In our study, a periareolar incision was used in 75 of the 85 cases (88.2\%). Although no direct relationship between the use of a periareolar incision and NAC necrosis was found in this study, it is nonetheless noteworthy that most patients underwent radical resection of the subareolar tissue through a periareolar incision.

BMI, smoking history, radiation therapy, and mastectomy volume were found in this study to have a significant relationship with NAC necrosis, which is in agreement with the general consensus regarding NAC necrosis. These variables are also risk factors for vascular insufficiency and flap necrosis. Other variables were not found to be significant in this study. Importantly, the incision method was not found to be a significant variable in this study, which was likely related to our use of periareolar incisions that did not extend more than halfway around the NAC.

Postoperative NAC necrosis is the complication of NSM that causes the most concern. Resection of the tissue located below the NAC causes injury to local perforators and a risk of ischemia in the NAC $[8,12]$. The onset of necrosis causes a deformity of the breast and depigmentation of the NAC. In addition, the possibility of treatment failure causes patient anxiety, and surgeons must provide patients with an alternative plan. In these cases, the use of banked skin is a good alternative. Using the skin-bank- ing technique means that surgeons do not need to explain to patients that a new skin donor site is necessary. The technique may also be indicated in high-risk patients with oncological nipple involvement. Although an intraoperative frozen biopsy may confirm the absence of malignancy, the results can change in the final pathological examination. In this case, surgeons can use the banked skin to replace the NAC in a NAC excision procedure. NAC replacement with banked skin can be performed under local anesthesia. In the case of the absence of necrosis or minimal necrosis, simple de-epithelialization and wound repair should be performed after observing NAC necrosis for one to two weeks. Hematoma removal, fat necrosis exploration, and wound revision can be performed simultaneously.

However, not all patients that present with NAC necrosis are available or willing to undergo a second operation. Hence, in this study, we reviewed the risk factors in order to identify highrisk patients.

In both the univariate and regression analysis, we were able to identify high-risk patients based on four risk factors: BMI, smoking history, radiation therapy, and mastectomy volume. With this definition, we were able to perform our skin-banking technique in only high-risk patients. Therefore, we suggest that patients who have two or more of the four risk factors we identified, or who show ischemic changes in the NAC intraoperatively, should be considered to be candidates for the skin-banking technique. In cases with a high risk of NAC necrosis, the skinbanking technique offers a good solution, and is not a form of over-treatment.

This study has certain limitations. It is a nonrandomized study; our study model was a retrospective review of medical charts, clinical records, and photographs. A single plastic surgeon performed the reconstructions, but two other surgeons performed the oncological breast surgery. The statistical power was weak because of the small sample size. Some variables examined in this study were subjective and thus could potentially be a source of bias. An objective analysis of patient and technical variables is needed in future studies. Despite these limitations, we were able to analyze the risk factors for NAC necrosis and obtained good results among high-risk patients through our skin-banking method. In the future, we anticipate prospective studies that construct an algorithm for the management of NAC necrosis.

\section{REFERENCES}

1. Spear SL, Hannan CM, Willey SC, et al. Nipple-sparing mastectomy. Plast Reconstr Surg 2009;123:1665-73.

2. Komorowski AL, Zanini V, Regolo L, et al. Necrotic complications after nipple- and areola-sparing mastectomy. World J 
Surg 2006;30:1410-3.

3. Endara M, Chen D, Verma K, et al. Breast reconstruction following nipple-sparing mastectomy: a systematic review of the literature with pooled analysis. Plast Reconstr Surg 2013; 132:1043-54.

4. Kim HR, Lim JS, Kim SM, et al. One-stage nipple and breast reconstruction following areola-sparing mastectomy. Arch Plast Surg 2013;40:553-8.

5. Tan BK, Chim H, Ng ZY, et al. Aesthetic design of skin-sparing mastectomy incisions for immediate autologous tissue breast reconstruction in asian women. Arch Plast Surg 2014; 41:366-73.

6. Gerber B, Krause A, Dieterich M, et al. The oncological safety of skin sparing mastectomy with conservation of the nipple-areola complex and autologous reconstruction: an extended follow-up study. Ann Surg 2009;249:461-8.

7. Kneubil MC, Lohsiriwat V, Curigliano G, et al. Risk of locoregional recurrence in patients with false-negative frozen section or close margins of retroareolar specimen in nipplesparing mastectomy. Ann Surg Oncol 2012;19:4117-23.

8. Moyer HR, Ghazi B, Daniel JR, et al. Nipple-sparing mastectomy: technical aspects and aesthetic outcomes. Ann Plast Surg 2012;68:446-50.

9. Carlson GW, Chu CK, Moyer HR, et al. Predictors of nipple ischemia after nipple sparing mastectomy. Breast J 2014;20: 69-73.

10. Colwell AS, Tessler O, Lin AM, et al. Breast reconstruction following nipple-sparing mastectomy: predictors of complications, reconstruction outcomes, and 5-year trends. Plast Reconstr Surg 2014;133:496-506.

11. Piper M, Peled AW, Foster RD, et al. Total skin-sparing mastectomy: a systematic review of oncologic outcomes and postoperative complications. Ann Plast Surg 2013;70:435-7.
12. van Deventer PV. The blood supply to the nipple-areola complex of the human mammary gland. Aesthetic Plast Surg 2004; 28:393-8.

13. Kovach SJ, Georgiade GS. The "banked” TRAM: a method to insure mastectomy skin-flap survival. Ann Plast Surg 2006; 57:366-9.

14. Liao EC, Labow BI, May JW Jr. Skin banking closure technique in immediate autologous breast reconstruction. Plast Reconstr Surg 2007;120:1133-6.

15. Park SW, Lee TJ, Kim EK, et al. Managing necrosis of the nipple-areola complex in breast reconstruction after nipplesparing mastectomy: immediate nipple-areola complex reconstruction with banked skin. Plast Reconstr Surg 2014;133: 73e-74e.

16. Reichl H, Hladik M, Wechselberger G. Skin banking: treatment option for native skin necrosis following skin-sparing mastectomy and previous breast irradiation. Microsurgery 2011;31:314-7.

17. Ahmed AK, Hahn DE, Hage JJ, et al. Temporary banking of the nipple-areola complex in 97 skin-sparing mastectomies. Plast Reconstr Surg 2011;127:531-9.

18. Gould DJ, Hunt KK, Liu J, et al. Impact of surgical techniques, biomaterials, and patient variables on rate of nipple necrosis after nipple-sparing mastectomy. Plast Reconstr Surg 2013; 132:330e-338e.

19. Dent BL, Small K, Swistel A, et al. Nipple-areolar complex ischemia after nipple-sparing mastectomy with immediate implant-based reconstruction: risk factors and the success of conservative treatment. Aesthet Surg J 2014;34:560-70.

20. Peled AW, Foster RD, Ligh C, et al. Impact of total skin-sparing mastectomy incision type on reconstructive complications following radiation therapy. Plast Reconstr Surg 2014; 134:169-75. 